\title{
Respostas Emocionais de Médicos aos Estímulos Afetivos do International Affective Picture System (IAPS)
}

\section{Emotional Responses of Doctors to the Affective Stimuli from the International Affective Picture System (IAPS)}

Laura Marques Castelhano ${ }^{I}$ Liliana Wahba

\section{PALAVRAS-CHAVE}

- Médicos.

- Emoções.

- Psicologia Médica.

\begin{abstract}
RESUMO
As emoções dos médicos têm um papel importante na relação com o paciente, pois podem interferir em suas atitudes, percepções e nos processos de julgamento e decisão. O objetivo desta pesquisa foi analisar a resposta emocional dos médicos aos estímulos do International Affective Picture System (IAPS). Este estudo utilizou o método quantitativo e avaliou 30 médicos, homens e mulheres de diversas especialidades, que dedicassem parte (ou a totalidade) de seu tempo ao atendimento clínico no consultório, que tivessem no mínimo dois anos de experiência após a conclusão da residência médica e que atuassem em medicina convencional (clínica médica). Os instrumentos utilizados na análise foram: o questionário sociodemográfico, para análise das variáveis; o IAPS, composto por imagens afetivas capazes de induzir estados emocionais; e o Self-Assessment Manikin (SAM), para classificação das respostas emocionais por meio de duas escalas: prazer e alerta. Os resultados mostraram que, quanto à percepção emocional, não houve diferença entre os resultados da amostra e da população geral, mas houve diferença na relação com as variáveis sociodemográficas - idade, tempo de formado, tempo médio de atendimento (consulta) e horas semanais dedicadas ao trabalho no consultório-, concluindo-se que médicos mais velhos, com mais tempo de formados e que ficam mais tempo em consulta com o paciente se sentiram mais impactados diante dos estímulos emocionais do que os médicos mais jovens, com menos tempo de formados e que ficam menos com o paciente em consulta. Os resultados também mostraram que os médicos que dedicam mais horas semanais ao trabalho no consultório perceberam os estímulos de forma menos prazerosa que os médicos que trabalham menos tempo no consultório e que dividem seu tempo com outras atividades. São necessárias novas pesquisas e estudos para o aprofundamento do tema das emoções e de sua influência na prática médica, considerando amostras maiores, em contextos distintos e novas variáveis.
\end{abstract}




\section{KEY-WORDS}

- Doctors.

- Emotions.

- Medical Psychology.

Recebido em: 20/11/18

Aceito em: 29/1/19
ABSTRACT

The emotions of doctors play an important role in their relationship with the patient as they can interfere with their attitudes, perceptions and in judgment and decision processes. The objective of the research was to analyze the emotional response of doctors to the stimuli of the International Affective Picture System (IAPS). This study used a quantitative method and evaluated 30 male and female doctors of various specialties, who dedicated all or some of their working hours to clinical care, had at least two years of experience after conclusion of medical residency and worked in conventional medicine (medical clinic). The instruments used for the analysis were: sociodemographic questionnaire to analyze the variables; the IAPS, composed of affective images capable of inducing emotional states and the Self-Assessment Manikin (SAM) to classify responses through two scales: pleasure and arousal. The results showed that in relation to emotional perception there was no difference between the sample results and the general population, but differences were found in the relation to sociodemographic variables: age, time since graduation, average consultation time and weekly hours devoted to work in the consulting room, concluding that older doctors, with more time since graduation and more time in consultation with the patient, felt more impacted by the emotional stimuli than younger doctors with less training time and less time with the patient in consultation. The results also showed that doctors who work more hours per week in the consulting room perceived the stimuli in a less pleasant way than doctors who spend less time in the consulting room and share their time with other activities. Further research and studies are needed to deepen the theme of emotions and their influence on medical practice, considering larger samples in different contexts and new variables.

\section{INTRODUÇÃO}

A compreensão dos fenômenos emocionais envolve uma problemática conceitual, já que, por ora, não existe consenso sobre a definição do que se entende por emoção. No campo da ciência, vêm ocorrendo tentativas que, ao privilegiarem o aspecto dinâmico da emoção, aumentam o diálogo entre as várias visões e enriquecem o desenvolvimento de teorias.

O ponto de convergência está na definição de que as emoções são programas de ações complexos, em grande parte automatizados, de difícil controle, que inter-relacionam a cognição, a comunicação, a motivação humana e a subjetividade ${ }^{1,2}$.

Segundo Damásio ${ }^{3}$, os processos cognitivos e emocionais são associados e dependentes. O autor desmitifica a ideia de uma "razão pura", em que as emoções são vistas como "intrusas" nos processos analíticos.

O aspecto dinâmico das teorias emocionais compreende que as emoções influenciam as vivências, as formas de ser dos indivíduos, têm um papel importante na dinâmica relacional e participam dos processos de escolha e tomada de decisão ${ }^{1,4}$.

Na prática médica, as emoções ocupam lugar de destaque, já que estão presentes na interação médico-paciente, influenciando relacionamentos e percepções. Apesar disto, o racionalismo e a tentativa constante de "eliminação" das emoções ainda estão presentes no discurso do médico ${ }^{5}$.

Desde o início da formação profissional, os médicos aprendem que as emoções e o sofrimento precisam ser negados; ao mesmo tempo em que precisam entrar em contato com questões dolorosas, eles aprendem o caminho da evitação. Nesta linha, Marco ${ }^{6}$ e Ramos-Cerqueira e Lima ${ }^{7}$ mostram que o médico tem pouco espaço para compartilhar ou expressar afetos, tendo que escondê-los por receio de ser "acusado" de ser muito frágil, sensível, e, portanto, "não servir para ser médico".

Os médicos, particularmente os mais jovens e recém-formados, desenvolvem comportamentos rígidos e estereotipados, com poucos recursos para lidar com o sofrimento, e transformam a exclusão das emoções, principalmente por conta dos mecanismos psicológicos de defesa, numa técnica necessária ao bom desempenho e atuação ${ }^{6,8,9}$.

Autores como Balint ${ }^{10}$, Groopman ${ }^{11}$, Croskerry ${ }^{12}$ e Mello Filho ${ }^{13}$ observam que os médicos, mesmo com os anos de prática, precisam lidar constantemente com o desconforto causado pelo impacto das emoções em sua profissão, já que as emoções sempre estarão presentes no trabalho do médico. 
Ser médico é lidar constantemente com o fato de que se está inserido numa relação. O médico não está isento de sentir, emocionar-se e desenvolver afetos em relação a seu paciente. Muito pelo contrário. É condição de qualquer relacionamento que isso se estabeleça. Como Balint ${ }^{10}$ assinala, a relação médico-paciente se desenvolve de forma mais efetiva quando os envolvidos na relação têm conhecimento do outro e de suas influências.

As emoções afloram, inevitavelmente, em qualquer relação, e o seu reconhecimento, em si e nos outros, e seu manejo apropriado contribuem para a construção de um campo emocional favorável ao desempenho da tarefa médica ${ }^{5}$.

Apesar de sua importância, estudos sobre a experiência emocional dos médicos recebem pouca atenção de pesquisadores. A medicina não se dedica a explorar esses fenômenos presentes nas relações e que têm papel central na construção do que é "ser médico".

A visão teórica que permite o estudo experimental da emoção tem suas bases em Wundt, um dos pioneiros na procura sistematizada do sistema afetivo. A dinâmica do sistema afetivo wundtiano corresponde a um espaço tridimensional representado pelas direções bipolares de variação, prazer-desprazer, alerta/tensão-relaxamento e ativação-desativação, fruto dos seus modos ou vias de expressão, quantificáveis em função da intensidade da sua manifestação ${ }^{14}$.

Segundo Bradley e Lang ${ }^{15}$, o trabalho empírico tem confirmado repetidamente que as categorias teóricas de Wundt estão presentes na organização dos julgamentos humanos para uma vasta gama perceptual de estímulos simbólicos.

Estímulos servem como indutores de respostas afetivas, cabendo aos sujeitos a tarefa de proceder aos julgamentos acerca do grau de prazer ou de desprazer (valência emocional), de alerta (intensidade) e grau de domínio que cada estímulo afetivamente sugere ${ }^{16}$.

Com tais diretrizes, o objetivo da pesquisa foi analisar a resposta emocional dos médicos aos estímulos do International Affective Picture System (IAPS) e as variáveis sexo, idade, tempo de formado, horas semanais dedicadas ao trabalho, horas semanais dedicadas ao trabalho no consultório, número de pacientes atendidos por dia e tempo médio de atendimento (consulta).

Foram formuladas duas questões norteadoras para este estudo: Existe diferença entre a resposta emocional dos médicos aos estímulos do IAPS e da população em geral? Há correlação entre a as respostas emocionais dos médicos aos estímulos do IAPS e as variáveis do estudo?

\section{MÉTODO}

A pesquisa utilizou a abordagem quantitativa e, como estratégia de investigação, o exame e a relação de variáveis medidas por instrumentos e analisados por procedimentos estatísticos ${ }^{17}$.

Participaram desta pesquisa 30 médicos, homens e mulheres, de diversas especialidades e que atendiam por convênio. Foram utilizados como critérios de inclusão: médicos que dedicassem parte (ou a totalidade) de seu tempo ao atendimento em consultório; que tivessem no mínimo dois anos de experiência após a conclusão da residência médica; que atuassem em medicina convencional (clínica médica). A coleta dos dados foi feita no próprio consultório de cada médico. $\mathrm{O}$ estudo foi realizado no período de abril de 2014 a fevereiro de 2015. Os instrumentos utilizados foram:

a) Questionário sociodemográfico: composto pelas variáveis sexo, idade, tempo de formado, horas semanais dedicadas ao trabalho, horas semanais dedicadas ao trabalho no consultório, número de pacientes atendidos por dia e tempo médio de atendimento (consulta);

b) International Affective Picture System (IAPS): composto por imagens afetivas que abordam diferentes eventos da vida e que são capazes de induzir uma gama de estados emocionais. Possui um banco de dados normatizado com imagens divididas em três categorias: agradáveis, desagradáveis e neutras. Para este estudo, foram selecionadas 36 imagens, que possuem como tema o relacionamento interpessoal e que foram divididas em 12 imagens desagradáveis, 12 agradáveis e 12 neutras ${ }^{18}$;

c) Self-Assessment Manikin (SAM): composto por três escalas - prazer, alerta e dominância -, classifica as respostas emocionais aos estímulos do IAPS. Optou-se por não utilizar a dimensão dominância devido a possíveis inconsistências relatadas em outras pesquisas. Cada escala possui cinco bonecos que expressam as gradações emocionais, que variam de 1 a 9. Os participantes marcam um " $x$ " sobre o item que mais se aproxima do estado emocional induzido pela imagem. A escala alerta mede a intensidade emocional, que, por meio de uma gradação induzida pelo estímulo do IAPS, pode ir de tenso e impactado a calmo e relaxado. A escala prazer mede a valência emocional, que, ao induzir a emoção por meio do estímulo do IAPS, pode ir de prazer e satisfação (agradável/positiva) a desprazer e insatisfação (desagradável/negativa) ${ }^{19}$.

A classificação e a análise das normas brasileiras foram realizadas por meio da comparação com o estudo original ${ }^{18}$, norte-americano. $\mathrm{O}$ estudo de validação ${ }^{20}$ certificou-se de que 
todos os coeficientes de correlação entre as normas brasileiras e americanas fossem significativos, fato que tem sido considerado para avaliação de sujeitos em diferentes países e culturas. A normatização para a população brasileira foi realizada por Ribeiro et al. ${ }^{20}$ e é empregada em diversos estudos sobre as emoções.

A utilização do IAPS e do SAM foi autorizada pelos autores. A pesquisa foi realizada no local de trabalho do médico (consultório). Os médicos foram convidados a participar da pesquisa, inicialmente, por meio de indicações de médicos conhecidos e depois pelo método "bola de neve", com indicações dos próprios entrevistados. Segundo Dewes ${ }^{21}$, esse método utiliza a rede de relacionamento dos participantes da amostra, que indicam outros indivíduos para participação no estudo.

Antes de iniciar a fase de pesquisa, foram realizados testes com dois médicos conhecidos para verificação dos instrumentos. Após essa etapa, os médicos participantes foram contatados, e, após a explicação dos objetivos da pesquisa e aceite do médico, foi agendada uma data para aplicação dos instrumentos e início da coleta.

O encontro obedeceu ao seguinte procedimento: explicação do objetivo, informações sobre a pesquisa e sequência da aplicação; informação sobre a confidencialidade e a possibilidade de se retirar da pesquisa a qualquer momento; assinatura do termo de consentimento e aplicação dos instrumentos. O tempo total para a aplicação dessa avaliação foi em média de 20 minutos.

Cada um dos instrumentos foi precedido de instrução. $\mathrm{O}$ primeiro instrumento aplicado foi o questionário sociodemográfico. Na sequência, a aplicação do IAPS foi feita com a apresentação das imagens ao participante, que registrou no bloco de respostas do $S A M$ a sua resposta emocional ao estímulo.

Para a análise quantitativa dos instrumentos aplicados, foram utilizados testes estatísticos e o programa SPSS.17 (Statistical Package for the Social Science), que descreve a amostra calculando suas medidas descritivas e realiza testes que relacionam as variáveis. O programa foi usado para a realização dos seguintes testes: teste de uma média; teste de duas médias para amostras independentes; análise de variância; e correlação de Pearson. Esta etapa incluiu os 30 médicos participantes da amostra. Cada médico recebeu um número com o qual foi identificado na pesquisa, e a análise de dados se baseou nessa identificação.

A análise quantitativa obedeceu à sequência: análise do sociodemográfico; análise do IAPS de acordo com as especificações a padronizações do teste, para as duas escalas do $S A M$, alerta e prazer; comparação do sociodemográfico com o IAPS.

A pesquisa foi aprovada pelo Comitê de Ética em Pesquisa da PUC-SP (processo no 126.632) e foram cumpridos os requerimentos éticos em pesquisa envolvendo seres humanos preconizados pela Resolução Conep 466/2012, do Conselho Nacional de Saúde, e pela Resolução CNS/MS 510/2016.

\section{RESULTADOS}

O tratamento estatístico dos dados quantitativos consistiu no cálculo de medidas descritivas da amostra adequadas ao nível de mensuração das variáveis envolvidas (média, desvio padrão, correlação de Pearson). Por outro lado, foram aplicados testes estatísticos para um nível de significância de 0,05. O critério para a aplicação desses testes também foi o de compatibilização com os tipos de variáveis.

A amostra contou com 30 médicos, 17 dos quais são do sexo masculino e 13 do sexo feminino; a média de idade foi de 44,6 anos, e a de tempo de formado, de 19,4 anos. A média de horas semanais dedicadas ao trabalho foi de 46 horas, e as dedicadas ao trabalho no consultório, de 32,8 horas. A média de pacientes atendidos por dia, no consultório, pelos médicos, foi de 21,9. O tempo médio de atendimento ao paciente no consultório foi de 26,8 minutos.

Os resultados do IAPS foram analisados para retratar as características da resposta emocional da amostra, comparando a média da amostra com o parâmetro da população brasileira.

A Tabela 1 apresenta a média da amostra para as escalas alerta e prazer comparada ao parâmetro da média da população.

\begin{tabular}{|c|c|c|c|c|c|c|c|}
\hline & $\mathbf{R e}$ & $\begin{array}{l}\text { nociona } \\
\text { n os pa }\end{array}$ & $\begin{array}{l}\text { lédicos } s \\
\text { s popul }\end{array}$ & no médi & $\begin{array}{l}\text { stra e } \\
\text { aulo, } 2\end{array}$ & ação & \\
\hline Escalas & $\mathbf{N}$ & Média & $\begin{array}{l}\text { Desvio } \\
\text { padrão }\end{array}$ & $\begin{array}{c}\text { Erro } \\
\text { padrão }\end{array}$ & $t$ & $\mathrm{p}$ & $\underset{\text { Parâmetro }}{\mu}$ \\
\hline Alerta & 30 & 4,80 & 0,95 & 0,173 & $-0,617$ & 0,542 & 4,91 \\
\hline Prazer & 30 & 5,13 & 0,36 & 0,066 & 1,857 & 0,074 & 5,01 \\
\hline Total & 30 & 4,97 & 0,44 & 0,08 & 0,095 & 0,925 & 4,96 \\
\hline
\end{tabular}

4 REVISTA BRASILEIRA DE EDUCAÇÃO MÉDICA

$49{ }_{43(3): 46-53 ; 2019}$ 
A comparação da amostra com os parâmetros da população do teste para as duas escalas foi realizada por meio do teste de uma média (teste $\mathrm{t}$ ), feito através do SPSS. Na escala alerta, a média da amostra foi de 4,80, e o parâmetro populacional, 4,91. Na escala prazer, a média da amostra foi de 5,13, e o parâmetro populacional, 5,01. Esses resultados indicam que os resultados da amostra são compatíveis com a população e, portanto, sem diferença significativa.

Procurou-se, num segundo momento, medir as relações entre a resposta emocional dos médicos e as variáveis sociodemográficas: sexo, idade, tempo de formado, horas semanais dedicadas ao trabalho, horas semanais dedicadas ao trabalho no consultório, número de pacientes/dia e tempo médio de atendimento (consulta).

Na Tabela 2, tem-se a comparação da média da amostra das duas escalas do IAPS, alerta e prazer, e a variável sexo.

\begin{tabular}{|c|c|c|c|c|c|c|c|}
\hline Respc & stas emo & 8 & $\begin{array}{r}\text { TAв } \\
\text { de mé } \\
\text { do a v } \\
\text { ão Pav }\end{array}$ & $\begin{array}{l}\text { LA } 2 \\
\text { licos ao } \\
\text { Iriável s } \\
\text { lo, } 2015\end{array}$ & $\begin{array}{l}\text { s estím } \\
\text { sexo. }\end{array}$ & los & APS, \\
\hline & Sexo & $\mathbf{N}$ & Média & $\begin{array}{l}\text { Desvio } \\
\text { padrão }\end{array}$ & $\begin{array}{c}\text { Erro } \\
\text { padrão }\end{array}$ & $t$ & $\mathbf{P}$ \\
\hline Jlorts & Masculino & 17 & 5,04 & 1,13 & 0,27 & & \\
\hline Alerta & Feminino & 13 & 4,49 & 0,54 & 0,15 & 1,634 & 0,057 \\
\hline & Masculino & 17 & 5,09 & 0,31 & 0,07 & 606 & 260 \\
\hline Prazer & & 13 & 5,18 & 0,43 & 0,12 & $-0,646$ & ,262 \\
\hline
\end{tabular}

A comparação da média da amostra das duas escalas do IAPS, alerta e prazer, e a variável sexo foi realizada por meio do teste de duas médias, demonstrando que não há diferença significativa entre homens e mulheres na relação com as escalas.

A seguir, na Tabela 3, verifica-se a correlação entre as escalas alerta e prazer do IAPS e as variáveis sociodemográficas.

A verificação da correlação entre as duas escalas, alerta e prazer, do IAPS e as variáveis sociodemográficas foi realizada utilizando-se o coeficiente de Pearson, estatística descritiva que mede o grau da correlação entre duas variáveis.

O coeficiente de Pearson entre idade e o alerta do IAPS foi igual a $+0,35$, o que indica uma relação positiva e moderada entre a idade e a escala alerta do IAPS. Isso significa que quanto maior a idade, maior a pontuação no alerta. Médicos mais velhos sentiram-se mais impactados e estimulados do que os médicos mais jovens diante dos estímulos emocionais.

Entre tempo de formado e o alerta do IAPS, o coeficiente de Pearson foi igual a $+0,32$, o que indica uma relação positiva

\begin{tabular}{|c|c|c|c|}
\hline \multicolumn{4}{|c|}{$\begin{array}{c}\text { TABELA } 3 \\
\text { Correlação entre as respostas emocionais de } \\
\text { médicos aos estímulos do IAPS e as variáveis } \\
\text { sociodemográficas. São Paulo, } 2015 .\end{array}$} \\
\hline Variável & & Alerta & Prazer \\
\hline \multirow{4}{*}{ Idade } & $\mathrm{r}$ & 0,35 & $-0,28$ \\
\hline & $\mathrm{p}$ & 0,031 & 0,065 \\
\hline & $\mathrm{N}$ & 30 & 30 \\
\hline & & Alerta & Prazer \\
\hline \multirow{4}{*}{ Tempo de formado } & $\mathrm{r}$ & 0,32 & $-0,24$ \\
\hline & $\mathrm{p}$ & 0,043 & 0,100 \\
\hline & $\mathrm{N}$ & 30 & 30 \\
\hline & & Alerta & Prazer \\
\hline \multirow{4}{*}{$\begin{array}{l}\text { Tempo total de trabalho } \\
\text { (horas semanais) }\end{array}$} & $\mathrm{r}$ & $-0,041$ & $-0,025$ \\
\hline & $\mathrm{p}$ & 0,415 & 0,448 \\
\hline & $\mathrm{N}$ & 30 & 30 \\
\hline & & Alerta & Prazer \\
\hline \multirow{4}{*}{$\begin{array}{c}\text { Tempo de trabalho no } \\
\text { consultório } \\
\text { (horas semanais) }\end{array}$} & $\mathrm{r}$ & 0,20 & $-0,40$ \\
\hline & $\mathrm{p}$ & 0,139 & 0,014 \\
\hline & $\mathrm{N}$ & 30 & 30 \\
\hline & & Alerta & Prazer \\
\hline \multirow{4}{*}{$\begin{array}{c}\text { Número de } \\
\text { pacientes/dia }\end{array}$} & $\mathrm{r}$ & 0,02 & 0,21 \\
\hline & $\mathrm{p}$ & 0,443 & 0,128 \\
\hline & $\mathrm{N}$ & 30 & 30 \\
\hline & & Alerta & Prazer \\
\hline \multirow{3}{*}{$\begin{array}{l}\text { Tempo médio de } \\
\text { atendimento (consulta) }\end{array}$} & $\mathrm{r}$ & 0,36 & $-0,204$ \\
\hline & $\mathrm{p}$ & 0,024 & 0,140 \\
\hline & $\mathrm{N}$ & 30 & 30 \\
\hline
\end{tabular}

e moderada. Isso significa que quanto maior o tempo de formado, maior a pontuação no alerta. Médicos com mais tempo de formados se sentiram mais impactados e estimulados do que os médicos mais jovens diante dos estímulos emocionais.

Houve correlação positiva e moderada $(+0,36)$ entre o tempo médio de atendimento e o alerta do IAPS, o que significa que quanto maior o tempo de consulta, maior o alerta. Quanto mais tempo o médico fica com o paciente durante o atendimento, mais impactado ele fica diante dos estímulos emocionais.

Houve correlação negativa e moderada $(-0,40)$ entre o tempo de trabalho (horas semanais trabalhadas) no consultório e a escala prazer do IAPS, o que significa que quanto mais horas semanais dedicadas ao trabalho no consultório, menor a pontuação em prazer.

Não houve correlação entre o total de horas semanais de trabalho e o número de pacientes atendidos por dia e as escalas alerta e prazer. 


\section{DISCUSSÃO}

Para responder à primeira questão do estudo - se existe diferença entre a resposta emocional dos médicos aos estímulos do IAPS e a da população em geral -, foi realizado teste de comparação da média da amostra do IAPS e os parâmetros populacionais, e não houve diferença significativa. Os médi$\cos$ da amostra não diferiram em relação à resposta emocional quando comparados com os resultados esperados.

Comparando os resultados obtidos com o que as pesquisas $^{6-8}$ apresentam como sendo a "fala" dos médicos diante do tema das emoções, pôde-se perceber que existe uma distância entre o que se revela no discurso, que mostra uma "figura distante" emocionalmente, e a forma como o médico reage diante dos impactos emocionais.

Segundo Balint ${ }^{10}$, os médicos apresentam um comportamento idealizado que reforça a imagem constituinte do que aprenderam em relação a "como ser médico" e, ao assumirem tal imagem, introjetam uma figura distante e sem afeto.

Ainda existe na medicina o modelo em que há uma cisão entre o pensamento racional e a resposta emocional. Essa dificuldade, também observada nas pesquisas de De $\mathrm{Marco}^{5}$, revela que, apesar dos avanços nas neurociências, a medicina ainda separa o pensamento do corpo, das vivências e das emoções.

Um médico pode desconhecer, ou tentar suprimir, sua resposta emocional, mas ela estará sempre presente na interação ${ }^{22}$.

Segundo Ofrii ${ }^{23}$, a interação médico-paciente é essencialmente humana, e, quando os seres humanos se conectam, as emoções tecem uma rede de interações. No caso da relação médico-paciente, cabe ao médico decidir como vai processar essas emoções.

Para responder à segunda questão - se há correlação entre as respostas emocionais dos médicos aos estímulos do IAPS e as variáveis do estudo -, foi investigada a correlação entre o IAPS e as variáveis sociodemográficas. Ao se observar internamente a amostra em função das distintas variáveis sociodemográficas, perceberam-se algumas tendências em relação à resposta emocional e às variáveis - idade, tempo de formado, horas semanais dedicadas ao trabalho no consultório e tempo médio de atendimento (consulta).

A seguir, são descritas três tendências que puderam ser notadas, sugerindo algumas hipóteses interpretativas.

\section{Primeira tendência}

Os resultados relacionados às variáveis idade e tempo de formado e a resposta emocional ao IAPS mostraram que médicos mais velhos, com mais tempo de formados, se sentiram mais impactados (alerta) diante dos estímulos emocionais.
A dimensão alerta se refere à intensidade, que varia de calma, relaxamento até excitação, e está associada à ativação da amígdala na sua relação com o hipocampo ${ }^{24}$.

Seja pela prontidão do médico a uma relação mais forte com o paciente ou por sua experiência e demanda emocional mais intensa em função do modo de consultar, os médicos mais velhos parecem mais sensíveis aos impactos emocionais.

\section{Segunda tendência}

Em relação à variável tempo médio de atendimento (consulta) e à resposta emocional ao IAPS, os resultados mostraram que quanto mais tempo o médico fica com o paciente durante o atendimento, mais impactado ele fica diante dos estímulos emocionais.

$\mathrm{O}$ alto alerta e o tempo maior com o paciente em consulta podem sugerir que os médicos estão mais sujeitos à intensidade das relações e que o tempo maior com o paciente os deixa mais suscetíveis aos estímulos e tensionados diante da carga emocional.

\section{Terceira tendência}

Em relação à variável horas semanais dedicadas ao trabalho no consultório e à resposta emocional ao IAPS, os dados mostraram que quanto mais horas semanais dedicadas ao trabalho no consultório, menor a pontuação em prazer.

Os médicos que ficaram mais horas semanais no consultório responderam ao estímulo de forma mais desagradável pela escala de prazer. Isto pode sugerir que o trabalho no consultório traria situações mais desagradáveis e de maior impacto na motivação. $\mathrm{O}$ baixo prazer pode sugerir baixo índice de satisfação.

A dimensão valência, conforme Lang ${ }^{25}$, refere-se a uma escala que varia do agradável ao desagradável. Informações de valência negativa ativam o sistema denominado aversivo e são responsáveis por comportamentos de luta, fuga ou congelamento.

\section{CONCLUSÕES}

Os médicos mais velhos, com mais tempo de formados e mais tempo de consulta se sentiram mais impactados diante dos estímulos emocionais. $\mathrm{O}$ estudo sugere que eles parecem ser mais sensíveis aos impactos emocionais do que os médicos mais jovens, com menos tempo de formados. Sugere também que os médicos que ficam mais tempo com o paciente durante o atendimento estão mais sujeitos à intensidade das relações.

Os médicos que dedicam mais horas semanais ao trabalho no consultório perceberam os estímulos de forma mais negativa e desagradável do que os médicos que trabalham menos 
tempo, o que sugere que o trabalho no consultório teria impacto na motivação.

Apesar das limitações do estudo por conta do tamanho e composição da amostra e pelo perfil dos médicos que atendem por meio de convênios, os resultados permitem obter informações importantes para o estudo das emoções dos médicos e abrem caminho para que novas pesquisas possam se aprofundar no tema das emoções e de sua influência na prática médica, considerando, talvez, contextos distintos e novas variáveis. Sugere-se que se ampliem as discussões sobre a resposta emocional do médico e o impacto nos processos perceptivos.

\section{REFERÊNCIAS}

1. Damásio A. E o cérebro criou o homem. São Paulo: Companhia da Letras; 2011.

2. Ledoux J. Cérebro emocional: os misteriosos alicerces da vida emocional. Rio de Janeiro: Objetiva, 2001.

3. Damásio A. O erro de Descartes: emoção, razão e o cérebro humano. 2. ed. São Paulo: Companhia da Letras; 2006.

4. Ekman P. A linguagem das emoções. São Paulo: Lua de Papel, 2011.

5. De Marco MA. Comunicação e relação. In: De Marco MA, Abud CC, Lucchese AC, Zimmermann VB. Psicologia Médica: abordagem integral do processo saúde-doença. Porto Alegre: Artmed; 2012. p.63-104.

6. Marco OLN. O estudante de medicina e a procura de ajuda. Rev Bras Educ Med. 2009; 33(3): 476-481.

7. Ramos-Cerqueira ATA, Lima MCP. A formação da identidade do médico: implicações para o ensino de graduação em medicina. Interface (Botucatu). 2002; 6(11):107-116.

8. Mascia AR, Silva FB, Lucchese AC, DeMarco MA, Martins MCFN, Martins LAN. Atitudes frente a aspectos relevantes da prática médica: estudo transversal randomizado com alunos de segundo e sexto anos. Rev Bras Educ Med. 2009; 33(1): 40-48.

9. Silva GSN. A construção do 'ser médico' e a morte: significados e implicações para a humanização do cuidado. São Paulo;2007. Tese [Doutorado em Medicina] - Universidade de São Paulo.

10. Balint M. O médico, seu paciente e a doença. 2. ed. Rio de Janeiro: Atheneu; 1988.

11. Groopman J. Como os médicos pensam. Rio de Janeiro: Agir; 2008

12. Croskerry P. Achieving quality in clinical decision making: cognitive strategies and detection of bias. Acad Emerg Med. 2002; 9(11): 1184-204.

13. Mello Filho J. Identidade médica: o normal e o patológico. In: Mello Filho J, org. Identidade médica: implicações históricas e antropológicas. São Paulo: Casa do Psicólogo; 2006. p.213-271.

14. Cardoso FMS. Estrutura e dinâmica do sistema afectivo das dimensões de avaliação às estruturas de acção-emoções: contributos para uma hermenêutica. Vila Real; 2008. Tese [Doutorado em Psicologia] - Universidade de Trás-os-Montes e Alto Douro.

15. Bradley M, Lang P. Measuring emotion: the self-assessment manikin and the semantic differential. J Behav Ther Exp Psychiatry.1994; 25(1): 49-59.

16. Mehrabian A, Russell EJ. Evidence for a three-factor theory of emotions. J Res Pers.1977; 11: 273-279.

17. Creswell JW. Projeto de pesquisa: métodos qualitativo, quantitativo e misto. 2. ed. Porto Alegre: Artmed; 2010.

18. Lang P, Bradley M, Cuthbert B. International affective picture system (IAPS): Instructional manual and affective ratings. 1997. [Technical report A-4]. The Center for Research in Psychology, University of Florida.

19. Pôrto WG, Bueno OFA, Bertolucci PHF. Manual de Instrução e Escores Afetivos: International Affective Picture System (IAPS) e Self-assessment manikin (SAM). [on line]. 2008. [acesso em 20 mai2012] Disponível em http:/ /www. unifesp.br/dpsicobio.

20. Ribeiro RL, Pompéia S, Bueno OFA. Normas brasileiras para o Internacional Affective Picture System (IAPS): comunicação breve. Rev. psiquiatr. Rio Gd. Sul. 2004;26(2): 190-194.

21. Dewes JO. Amostragem em Bola de Neve e Respondent-Driven Sampling: uma descrição dos métodos. Porto Alegre; 2013. Monografia [Bacharel em Estatística] - Universidade Federal do Rio Grande do Sul.

22. Meier DE, Back AL, Morrison RS. The inner life of physicians and care of the seriously ill. JAMA. 2001; 286(23): 3007-14.

23. Ofri D. What doctors feel: how emotions affect the practice of medicine. Boston: Beacon Press; 2014.

24. Dolcos F, Kragel P, Wang L, McCarthy G. Role of the inferior frontal cortex in coping with distracting emotions. Neuroreport. 2006; 17(15):1591-1594.

25. Lang PJ. The emotion probe: studies of motivation end attention. Am. Psychol. 1995; 50(5):372-385. 


\section{CONTRIBUIÇÃO DOS AUTORES}

Todos os autores participaram da concepção do trabalho, análise de dados, revisão bibliográfica e redação do manuscrito, aprovando a versão final.

\section{CONFLITO DE INTERESSES}

Os autores declaram não possuir conflitos de interesses.

\section{ENDEREÇO PARA CORRESPONDÊNCIA}

Pontifícia Universidade Católica de São Paulo. Rua Monte Alegre, 984 - Perdizes - São Paulo - SP CEP: 05014-901. Secretaria da FACHS. E-mail: lmcastelhano@pucsp.br 